Chirurg 2021 $\cdot 92: 630-639$

https://doi.org/10.1007/s00104-021-01448-z

Angenommen: 31. Mai 2021

Online publiziert: 21. Juni 2021

(c) Der/die Autor(en) 2021

\section{Ökonomische Auswirkung der COVID-19-Pandemie in der Allgemein- und Viszeralchirurgie}

\author{
Johannes Binder ${ }^{1}$ Maximilian Brunner' $\cdot$ Matthias Maak $^{1,2} \cdot$ Axel Denz $^{1}$. \\ Georg F. Weber ${ }^{1} \cdot$ Robert Grützmann ${ }^{1,2} \cdot$ Christian Krautz $^{1}$ \\ ${ }^{1}$ Klinik für Allgemein- und Viszeralchirurgie des, Universitätsklinikum Erlangen, Erlangen, Deutschland \\ ${ }^{2}$ Chirurgische Abteilung, Kreiskrankenhaus St. Anna Höchstadt/Aisch, Höchstadt a. d. Aisch, Deutschland
}

Zusammenfassung

\section{Hintergrund und Fragestellung}

Aufgrund hoher Infektionszahlen mit zum Teil schweren Krankheitsverläufen hat die COVID-19(",coronavirus disease 2019")Pandemie bereits früh zu erheblichen Schwierigkeiten in den Gesundheitssystemen einiger Länder geführt. Zur Vermeidung der drohenden Überlastung des Deutschen Gesundheitssystems wurden alle Krankenhäuser im März 2020 dazu aufgefordert, planbare Operationen und Eingriffe zu verschieben. Hierdurch konnten zusätzliche Kapazitäten und Ressourcen zur Behandlung von COVID-19Patienten freigesetzt werden [3].

Speziell im Fokus der Kapazitätssteigerung und Ressourcenbündelung waren hierbei die Intensivstationen. In Bayern trat im Zuge dessen am 19.03.2020 eine Allgemeinverfügung auf Grundlage von § 28 Abs. 1 Satz 1 des Infektionsschutzgesetzes (IfSG) in Kraft. Demnach hatten unterschiedliche Einrichtungen der Patientenversorgung „bis auf Weiteres alle planbaren Behandlungen zurückzustellen oder zu unterbrechen", um so zusätzliche materielle und personelle Ressourcen zu schaffen. Dennoch musste sichergestellt werden, dass es bei den weiterhin notwendigen Behandlungen zu keiner Einschränkung der Versorgungsqualität kam.

Neben den sich daraus ergebenden medizinischen Herausforderungen traten auch mögliche wirtschaftliche Risiken in den Fokus. Als Grundlage der Ab-

Hintergrund: Bereits während der ersten Welle der COVID-19-Pandemie wurden die deutschen Krankenhäuser dazu aufgefordert, ihre elektiven Operationskapazitäten einzuschränken, um eine Überlastung des Gesundheitswesens abzuwenden. Im März 2020 wurden mit dem COVID-19-Krankenhausentlastungsgesetz finanzielle Hilfen zum Ausgleich dieser Einschränkungen vereinbart. Die Auswirkungen dieser Maßnahmen wurden in dieser Studie regional untersucht.

Material und Methoden: Es wurden die Leistungsdaten und Erlöskennzahlen der Klinik für Allgemein- und Viszeralchirurgie des Universitätsklinikums Erlangen (UKER) und der Chirurgischen Abteilung des Kreiskrankenhauses St. Anna Höchstadt/Aisch (KKH) im Zeitraum 01.04. bis 30.06.2019 mit denen des Jahres 2020 verglichen.

Ergebnisse: Es zeigte sich eine deutliche Reduktion der Bettenauslastung und der Fallzahlen stationär behandelter Patienten. Letztere sanken um 20,06\% im UKER bzw. $60,76 \%$ im KKH. Nichtonkologische elektive Operationen nahmen um 33,04\% (UKER) bzw. 60,87\% (KKH) ab. Die Anzahl der Notfalleingriffe blieb am UKER unverändert und verringerte sich am KKH um 51,58\%.Die Erlöse aus DRG („diagnosis-related groups") sanken um 22,12\% (UKER) und $54 \%$ (KKH). Nach Berücksichtigung der Ausgleichszahlungen und Einsparungen aus variablen Sachkosten verzeichnete das UKER einen Verlust von -3,87\%, das KKH erreichte hingegen einen positiven Erlöseffekt von $6,5 \%$.

Diskussion: Die nichtselektive Einschränkung des elektiven Operationsbetriebs beeinflusste die Patientenversorgung und die Erlöskennzahlen an beiden Standorten signifikant. In Bezug auf die Schaffung von Intensivkapazitäten erscheint diese ungezielte Maßnahme jedoch nicht effizient. Zudem führte die einheitliche Freihaltepauschale zu einer unausgeglichenen Verteilung der finanziellen Hilfen zwischen den untersuchten Kliniken.

\title{
Schlüsselwörter
}

Krankenhausentlastungsgesetz - Coronavirus · Chirurgie · DRG-Abrechnungsdaten . Behandlungsqualität

rechnung stationärer Leistungen dient in Deutschland das G-DRG(„diagnosis-related groups")-System. Die Entscheidung zur Einschränkung des Normalbetriebes hatte somit zwangsläufig finanzielle Auswirkungen auf eine bereits stark im Wettbewerb stehende Krankenhauslandschaft zur Folge. Schätzungen, die während der Vorbereitung dieser Maßnahmen z.B. durch die Diakonie Deutschland und dem Deutschen Evangelischen Krankenhausverband (DEKV) durchgeführt wurden, gingen in einer Modellrechnung von Ausfällen in Millionenhöhe aus [10, 11]. 


\section{Infobox 1}

Übersicht zu den finanziellen Hilfen aus dem COVID-19-Krankenhausentlastungsgesetz (Stand 27.03.2020)

- Freihaltepauschale in Höhe von $560 €$

- Bonus für zusätzliche Intensivbetten in Höhe von $50.000 €$

- Mehrkosten für Schutzausrüstung in Höhe von $50 €$ pro Patient

- Vorläufiger Pflegeentgeltwert erhöht auf $185 €$

- Aussetzung des Fixkostendegressionsaufschlags

- Verringerung der Prüfquote auf 5\%

- Aussetzung der Abschläge bei Abrechnungskorrekturen

Demnach ergäben sich für ein Krankenhaus mit 300 bis 400 Betten bei einem Wegfall von ca. $50 \%$ der nicht dringlichen Behandlungen ein jährlicher Verlust von 12 Mio. $€$. Die Bundesregierung kündigte daher eine umfassende finanzielle Unterstützung an.

Am 27.03.2020 trat das Gesetz zum Ausgleich COVID-19-bedingter finanzieller Belastungen der Krankenhäuser und weitererGesundheitseinrichtungen („COVID-19Krankenhausentlastungsgesetz") in Kraft [6]. Das Gesetz sah umfangreiche Maßnahmen vor (s. DInfobox 1). Unter anderem sollte eine bundeseinheitliche, tagesbezogene Freihaltepauschale in Höhe von $560 €$ für jedes freigehaltene Bett (in Bezug auf die durchschnittliche Belegung des Vorjahres) die Einnahmeausfälle der Krankenhäuser abfedern. Diese finanzielle Unterstützung war zunächst für den Zeitraum vom 16.03.2020 bis 30.09.2020 vorgesehen.

Die vorgelegten Maßnahmen unterlagen bereits zum Zeitpunkt ihrer Einführung einer breiten Diskussion. Dabei wurde vor allem die Zielgenauigkeit bzw. die Verteilungsungerechtigkeit der bundeseinheitlichen Freihaltepauschale kritisiert. Letztere wurde schließlich ab dem 13.07.2020 durch differenzierte Beträge in fünf Stufen zwischen 360 bis $760 €$ abgelöst. Nichtdestotrotz bleibt eine pauschalierte Zahlung vor dem Hintergrund höchst variabler Kostenstrukturen der Krankenhäuser allenfalls ein kurzfristiges Mittel. Auf der Krankenhausebene bleiben zudem die Unterschiede zwischen Fachrichtungen unberücksichtigt, was langfristig zu betriebswirtschaftlichen
Spannungen innerhalb der Krankenhäuser führen kann.

Wie stark sich die Einschränkung des Regelbetriebes auf die Erlössituation in der Chirurgie auswirkt und welche Unterschiede in Bezug auf Krankenhausgröße, Behandlungsspektrum und Fallzahlen bestehen, ist weiterhin nur unzureichend beantwortet. Vor dem Hintergrund der zweiten Pandemiewelle und der weiterhin hohen Anzahl an Infektionen (2.134.936) und Todesfällen (51.870) in Deutschland (Stand 24.02.2020) sind diese Fragen relevanter denn je [20].

Ziel dieser Studie war es, die Auswirkungen der Einschränkung des Normalbetriebs und der finanziellen Hilfen des COVID-19-Krankenhausentlastungsgesetzes im Hinblick auf die Leistungsdaten und die Erlössituation in der Allgemein- und Viszeralchirurgie im direkten Vergleich zweier Kliniken aus unterschiedlichen Krankenhausversorgungsstufen zu analysieren.

\section{Studiendesign und Untersuchungsmethoden}

Als Datengrundlage dienten bei dieser retrospektiven Studie die G-DRG-Abrechnungsdaten aller stationären Fälle der Klinik für Allgemein- und Viszeralchirurgie des Universitätsklinikums Erlangen (UKER) sowie der Chirurgischen Abteilung des Kreiskrankenhauses Höchstadt (KKH). Das Universitätsklinikum Erlangen ist ein Krankenhaus der Maximalversorgung, während das Kreiskrankenhaus Höchstadt als Krankenhaus der Grundversorgung eingestuft ist. Die von der Politik vorgegebenen Einschränkungen wurden in den jeweiligen Krankenhäusern ab dem 19.03.2020 umgesetzt. Die fixe Pauschale für somatische Krankenhäuser wurde durch die COVID-19-AusgleichszahlungsÄnderungs-Verordnung (AusglZÄV) vom 13.07.2020 durch fünf Ausgleichsstufen abgelöst [7]. Da die abrechnungsrelevanten Daten nicht tagesaktuell, sondern lediglich monatsweise zur Verfügung gestellt werden konnten, wurde für diese Studie als Beobachtungszeitraum der 01.04.2020 bis 30.06.2020 gewählt. Als Vergleichszeitraum dienen die Zahlen des entsprechenden Zeitraums im Jahr 2019. Die Daten eignen sich insofern zum direkten Vergleich, da die Versorgung chirurgischer Patienten des Kreiskrankenhauses Höchstadt, im Rahmen einer Kooperation, unter ärztlicher Leitung der Chirurgischen Klinik des Universitätsklinikums Erlangen erfolgt und daher gleiche Standards gelten.

Als Referenzzeitpunkt zum Einschluss wurde das Aufnahmedatum gewählt. Patienten, die über den Beobachtungszeitraum hinaus stationär behandelt wurden, wurden nicht ausgeschlossen. Die Bettenauslastung wurde auf Monatsbasis berechnet, wobei die tatsächliche mit der maximalen Bettenbelegung ins Verhältnis gesetzt wurde, welche sich aus dem Produkt der insgesamt verfügbaren Betten und der Anzahl der Kalendertage im Berichtszeitraum errechnet. Als Grundlage dienten die Planbetten der Normalstation, ohne zugehörige Intensivkapazitäten.

Die durchgeführten Operationen wurden in Operationskategorien (z. B. Adipositaschirurgie, hepatobiliäre Chirurgie etc.) zusammengefasst (s. Anhang - Tab. 1). So wurden beispielsweise proktologische Operationen in der Kategorie „Proktologie" zusammengefasst, wohingegen einzelne häufig durchgeführte Operationen (z.B. Cholezystektomie) eine eigene Kategorie bekamen. Zudem erfolgte eine Stratifizierung nach Alter, Geschlecht, Dringlichkeit (elektiv/Notfall), Aufnahmeart (Wiederaufnahme ja/nein), Indikationsart (onkologisch/nichtonkologisch), Zugangsweg (offen/laparoskopisch/roboterassistiert/ retroperitoneoskopisch), Verweilweildauer und Art der Entlassung (regulär/externe Verlegung/gestorben). Im Weiteren wurden der Case Mix (CM), der Case-Mix-Index (CMI), die Erlöse und die UGDV(untere Grenzverweildauer)-Abschläge berechnet. Letztere sind eine Kennzahl, die sich durch Absenkung der bundeseinheitlichen Prüfquote des Medizinischen Dienstes der Krankenversicherung (MDK) gemäß dem COVID-19-Krankenhausentlastungsgesetz erlösrelevant ändern könnte. Obwohl die Einhaltung der Verweildauern den MDK per se nicht zur Abrechnungsprüfung berechtigt, werden insbesondere Krankenhausbehandlungen an der unteren Grenzverweildauer häufig geprüft [14, 24].

Um eine Vergleichbarkeit der Erlöse zu gewährleisten, wurden die Erlöse im 
Tab. 1 Vergleich der stationären Fälle zwischen den Beobachtungszeiträumen 2019 und 2020 an der Chirurgischen Klinik Erlangen

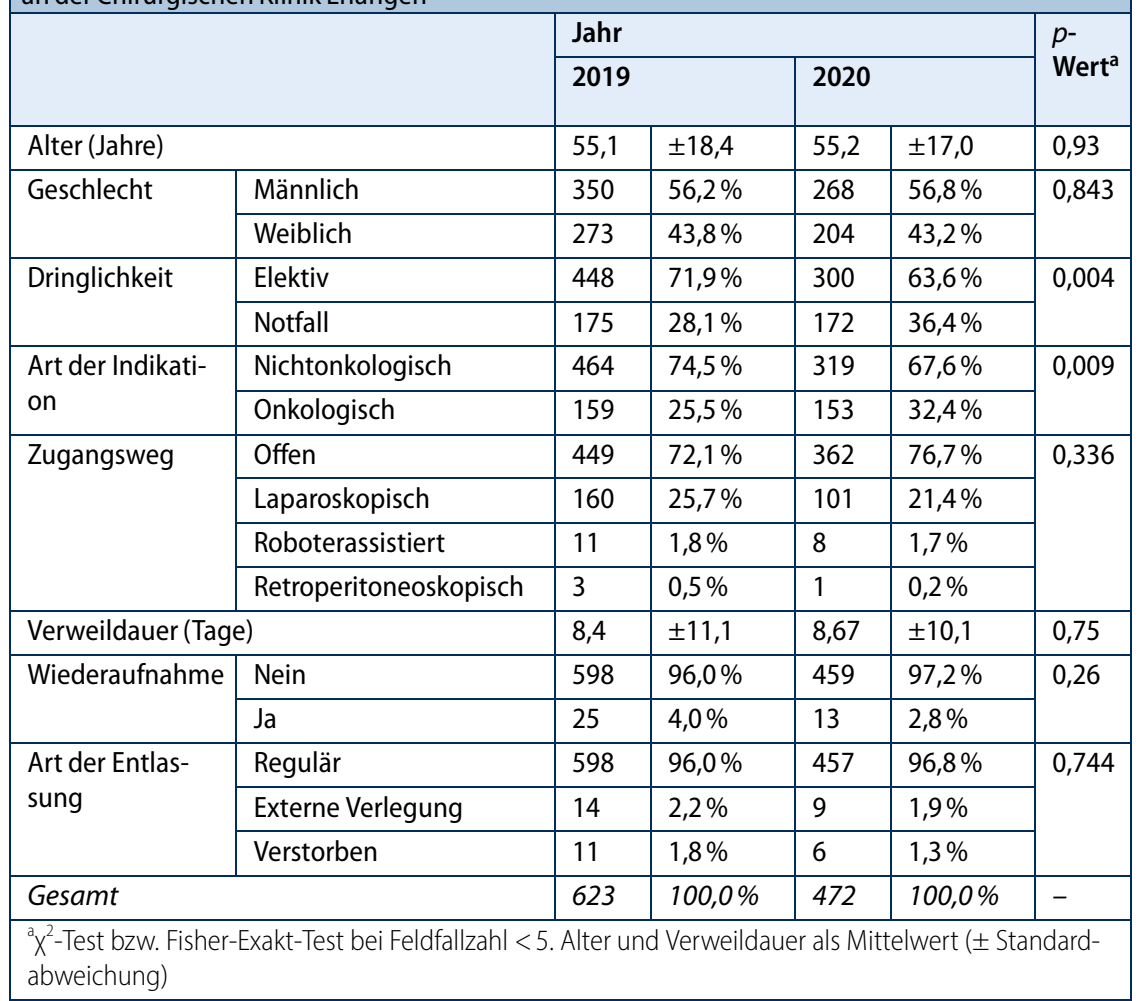

Jahr 2020 ohne Preissteigerung der Entgelte berechnet. Daher wurde für 2019 und 2020 der gleiche Landesbasisfallwert $(3560,54 €)$ verwendet. Zudem wurden die für das Jahr 2019 zugrunde liegenden Daten unter Verwendung des sog. Übergangsgrouper 2019 bis 2020 um die Pflegepersonalkosten bereinigt, da diese ab 2020 nicht mehr im DRG-System abgebildet werden.

Zur Berechnung der Höhe der Freihaltepauschalen wurde die Anzahl der im Jahresdurchschnitt 2019 voll- oder teilstationär behandelten Patienten auf einen Zeitraum von drei Monaten kondensiert und hiervon die Zahl der Belegungstage im Untersuchungszeitraum 2020 abgezogen. Eine positive Differenz wurde mit der tagesbezogenen Pauschale von $560 €$ multipliziert. Die Zuschläge in Höhe von $50 €$ für zusätzliche Schutzausrüstung pro Fall wurden ebenfalls mit der entsprechenden Fallzahl multipliziert. Die Erhöhung des Pflegeentgeltwerts auf $185 €$ wurde ebenfalls nicht in die Analyse eingeschlossen, da die Ausgliederung der Pflegekosten erst im Jahr 2020 wirksam wurde und für das Jahr 2019 keine Definition der Pflegepersonalkosten bzw. keine Vereinbarung der Pflegebudgets vorlagen. Die Aufhebung des Fixkostendegressionsabschlages für das Jahr 2020 wurde nicht berücksichtigt.

Zum Zeitpunkt der Erhebung lagen die Kostendaten beider Krankenhäuser nicht abschließend vor, somit musste auf eine detaillierte Kosten- und Erlösgegenüberstellung verzichtet werden. Für die Fixkosten wurden daher für das Jahr 2020 angenommen, dass sich diese zu 2019 nicht verändert haben. Für die variablen Sachkosten wurde pauschal ein Betrag in Höhe von $15 \%$ der Entgelte je Fall zugrunde gelegt. Dieses Vorgehen wurde bereits von Augurzky et al. angewandt [1]. Basierend auf diesen vereinfachten Annahmen wurde die Erlössituation anschließend kalkuliert. Hierbei setzen sich die Bruttoerlöse aus den Erlösen ohne Pflegekosten zuzüglich der Summe aus der Freiheitspauschale und den Zusatzentgelten für Schutzmaßnahmen zusammen. Die Nettoerlöse ergeben sich aus den Bruttoerlösen zuzüglich der Differenz aus den variablen Sachkosten. Bei Einsparungen aufgrund nicht angefallener variabler Sachkosten liegt somit ein positiver Erlöseffekt vor.
Die o.g. Daten wurden bei normalverteilten, kontinuierlichen Daten mittels ungepaartem T-Test analysiert. Binäre oder kategoriale Daten wurden mittels $x^{2}$-Test oder dem Exakten Test nach Fisher analysiert. Die Daten wurden als absolute und relative Häufigkeiten bzw. als Mittelwerte mit Standardabweichungen dargestellt. Das Signifikanzniveau (a) wurde auf $5 \%$ festgelegt (zweiseitig).

\section{Ergebnisse}

Auswirkungen auf die Bettenauslastung, Fallzahlen und Behandlungsqualität

Die Chirurgische Klinik des Universitätsklinikums Erlangen hatte in den Jahren 2019 und 2020 insgesamt 63 Planbetten zur Verfügung. Für das Kreiskrankenhaus Höchstadt (KKH) wurden 5 Planbetten für die Allgemein- und Viszeralchirurgie als Berechnungsgrundlage verwendet. Hierbei muss beachtet werden, dass die tatsächliche Anzahl an Planbetten in Höchstadt tatsächlich höher wäre, aufgrund von Umbauarbeiten diese jedoch nicht voll genutzt werden konnten. Zudem liegt der Schwerpunkt der chirurgischen Versorgung im Bereich der Unfallchirurgie und Orthopädie. Im Vergleich zu den Vorjahresmonaten ist die Bettenauslastung in Erlangen von $93 \%$ auf $74 \%$, in Höchstadt von $79 \%$ auf $36 \%$ gesunken. Die Fallzahlen stationär behandelter Patienten sanken im Betrachtungszeitraum von $718 \mathrm{im}$ Jahr 2019 auf $574 \mathrm{im}$ Jahr 2020 (UKER) bzw. von 79 auf 31 (KKH).

Insgesamt wurden im Betrachtungszeitraum 2019 am UKER 623 und am KKH 77 Operationen durchgeführt. Demgegenüber war 2020 in beiden Krankenhäusern ein deutlicher Rückgang der Operationszahlen zu verzeichnen. Am UKER kam es zu einem Rückgang um 24,24\% auf 472 Operationen, am KKH sogar um 54,55\% auf 35. Die Entwicklung der Fallzahlen entsprechend den Operationskategorien ist in - Tab. 1 im Anhang dargelegt. Entgegen dem Gesamttrend fanden sich in einzelnen Kategorien Ausreißer (z.B. „multiviszerale Resektionen" $+100 \%$ ), welche jedoch auf niedrige Fallzahlen zurückzuführen sind.

Die Stratifizierung der Eingriffe nach Dringlichkeit ergab in Erlangen eine signifikante Abnahme der elektiven Operationen 
Tab. 2 Vergleich der stationären Fälle zwischen den Beobachtungszeiträumen 2019 und 2020 an der Chirurgischen Klinik Höchstadt

\begin{tabular}{|c|c|c|c|c|c|c|}
\hline & \multicolumn{4}{|l|}{ Jahr } & \multirow{3}{*}{$\begin{array}{l}p- \\
\text { Wert }^{\mathrm{a}}\end{array}$} \\
\hline & & \multicolumn{2}{|c|}{2019} & \multicolumn{2}{|c|}{2020} & \\
\hline \multicolumn{2}{|l|}{ Alter (Jahre) } & 54,1 & $\pm 17,4$ & 58,9 & $\pm 18,6$ & \\
\hline \multirow[t]{2}{*}{ Geschlecht } & Männlich & 51 & $66,2 \%$ & 21 & $60,0 \%$ & \multirow[t]{2}{*}{0,523} \\
\hline & Weiblich & 26 & $33,8 \%$ & 14 & $40,0 \%$ & \\
\hline \multirow[t]{2}{*}{ Dringlichkeit } & Elektiv & 54 & $70,1 \%$ & 27 & $77,1 \%$ & \multirow[t]{2}{*}{0,442} \\
\hline & Notfall & 23 & $29,9 \%$ & 8 & $22,9 \%$ & \\
\hline \multirow{2}{*}{$\begin{array}{l}\text { Art der Indikati- } \\
\text { on }\end{array}$} & Nichtonkologisch & 77 & $100,0 \%$ & 35 & $100,0 \%$ & \multirow[t]{2}{*}{-} \\
\hline & Onkologisch & - & - & - & - & \\
\hline \multirow[t]{4}{*}{ Zugangsweg } & Offen & 53 & $68,8 \%$ & 31 & $88,6 \%$ & \multirow[t]{4}{*}{0,033} \\
\hline & Laparoskopisch & 24 & $31,2 \%$ & 4 & $11,4 \%$ & \\
\hline & Roboterassistiert & 0 & $0,0 \%$ & 0 & $0,0 \%$ & \\
\hline & Retroperitoneoskopisch & 0 & $0,0 \%$ & 0 & $0,0 \%$ & \\
\hline \multicolumn{2}{|c|}{ Verweildauer(Tage) } & 5,8 & $\pm 8,1$ & 10,0 & $\pm 12,4$ & 0,073 \\
\hline \multirow[t]{2}{*}{ Wiederaufnahme } & Nein & 73 & $94,8 \%$ & 30 & $85,7 \%$ & 0,135 \\
\hline & $\mathrm{Ja}$ & 4 & $5,2 \%$ & 5 & $14,3 \%$ & - \\
\hline \multirow{3}{*}{$\begin{array}{l}\text { Art der Entlas- } \\
\text { sung }\end{array}$} & Regulär & 76 & $98,7 \%$ & 32 & $91,4 \%$ & \multirow[t]{3}{*}{0,09} \\
\hline & Externe Verlegung & 1 & $1,3 \%$ & 3 & $8,6 \%$ & \\
\hline & Verstorben & 0 & $0,0 \%$ & 0 & $0,0 \%$ & \\
\hline \multicolumn{2}{|l|}{ Gesamt } & 77 & $100,00 \%$ & 35 & $100,00 \%$ & - \\
\hline
\end{tabular}

$(-33,04 \%)$ bei fast unveränderter Anzahl an Notfalleingriffen $(-1,71 \%)$, was zu einer signifikanten Zunahme des Notfallanteils im Jahr 2020 führte ( $p<0,004 ;$ - Tab. 1). Gleichfalls sank die Anzahl der onkologischen Eingriffe im Vergleich zu den nichtonkologischen Operationen deutlich weniger $(-3,77 \%)$.

Surrogatparameter für die Behandlungsqualität wie z.B. Verweildauer, Wiederaufnahmerate und Mortalitätsrate unterlagen am UKER keinen signifikanten Veränderungen. Am Grundversorger (KKH) kam es zu einem deutlichen Rückgang von Elektiv- und Notfalleingriffen (-60,9\% vs. $-51,5 \%)$, sodass sich der relative Anteil zwischen den Dringlichkeitsstufen nicht änderte ( $p=0,442$; Dab. 2). Onkologische Operationen werden u.a. aufgrund der Nähe zum Maximalversorger (Darmkrebs- und Pankreaskarzinomzentrum, Sarkomzentrum, Leberzentrum und Hautkrebszentrum Erlangen) in Höchstadt nicht durchgeführt, womit der Vergleich zu nichtonkologischen Eingriffen entfiel. Interessanterweise wurden im Beobachtungszeitraum 2020 signifikant mehr offene und weniger laparoskopische Eingriffe im Vergleich zum Vorjahreszeitraum durchgeführt $(p=0,033)$. Analog zum Maximalversorger wurden die Surrogatparameter für die Behandlungsqualität (Verweildauer, Wiederaufnahmerate und Mortalitätsrate) in Höchstadt ebenfalls nicht durch die Einschränkungen des Normalbetriebes beeinflusst.

\section{Auswirkungen auf die Erlös- kennzahlen}

Die Einschränkung des Normalbetriebes hatte in beiden Kliniken teils erhebliche Auswirkungen auf die Erlöskennzahlen (-Abb. 1). Aufgrund des geringeren $\mathrm{Pa}$ tientenaufkommens nahmen der Case Mix und die Erlöse statistisch signifikant ab. Dieser Einbruch war in Erlangen mit $-22,1 \%$ geringer als am KKH $(-54 \%$; - Tab. 3). Der CMI des UKER zeigte für den Monat Mai einen leichten Anstieg, wohingegen der CMI im Juni wieder sank. Ein ähnlicher Anstieg wurde zu Beginn der Einschränkungen bereits an einem anderen universitären Maximalversorger beobachtet [8]. Im Mittel kam es jedoch zu keiner statistisch signifikanten Änderung der Fallschwere in Erlangen (CMI: 1,66 vs. 1,69; $p=0,43)$. Der CMI am KKH nahm von 0,76 im Jahr 2019 auf 0,89 im Jahr 2020 zu. Diese Veränderung war jedoch nicht statistisch signifikant $(p=0,59)$.

Die Höhe der UGVD-Abschläge nahm am UKER zwar signifikant ab $(p=0,01)$, in Relation zum DRG-Erlös ergab sich jedoch keine wesentliche Änderung (2019:2,77\% vs. 2020: 2,76\%). Mögliche Einspareffekte waren hier mit $211,95 €$ vernachlässigbar. Demgegenüber änderte sich die absolute Höhe der UGVD-Abschläge für das KKH nicht signifikant. Es fand sich aber in Relation zum DRG-Erlös ein deutlicher Rückgang (2019: 11,56\% vs. 2020: 6,97\%), mögliche Einsparungen durch gesunkene UGVD-Abschläge belaufen sich auf $4484,41 €$.

\section{Veränderung der Erlössituation durch finanzielle Hilfen}

Unter Einbeziehung der Freihaltepauschalen sowie der Zulagen für Schutzausrüstung änderte sich die Erlössituation deutlich zugunsten des Grundversorgers (• Tab. 3). Während die Bruttoerlöse des UKER um 7,2 \% sanken, zeigte sich in Höchstadt nur noch ein Rückgang von 1,6\%. Nach Berücksichtigung der Einsparungen aus variablen Sachkosten (Nettoerlöse) ergab sich sogar ein positiver Erlöseffekt von 6,5\% für das $\mathrm{KKH}$, während die Nettoerlöse des UKER noch 3,9\% unter den Vorjahreszahlen lagen. Um den Effekt der abgestuften Freihaltepauschalen zu simulieren, wurden die Bruttoerlöse mit der jeweiligen abgestuften Freihaltepauschale erneut berechnet. Hierbei fand sich für das UKER ein positiver Erlöseffekt von $1,17 \%$ und ein Erlösrückgang zum Vorjahr von $-2,8 \%$ für das $\mathrm{KKH}$.

\section{Diskussion}

Die von der Politik beschlossenen Einschränkungen wurden an beiden betrachteten Krankenhäusern umgesetzt. Die Auswirkungen auf die Fallzahlen sowie auf die Erlössituation waren im Vergleich sehr unterschiedlich. So sind am KKH die Fallzahlen überproportional stark gesunken. Besonders hervorzuheben ist hier die Abnahme der Notfalleingriffe um 51,6\%. In diesem Zusammenhang könnte zu befürchten sein, dass es hier eventuell zu Einschränkungen in der Notfallbehandlung gekommen ist. Slagmann et al. zeigten, 


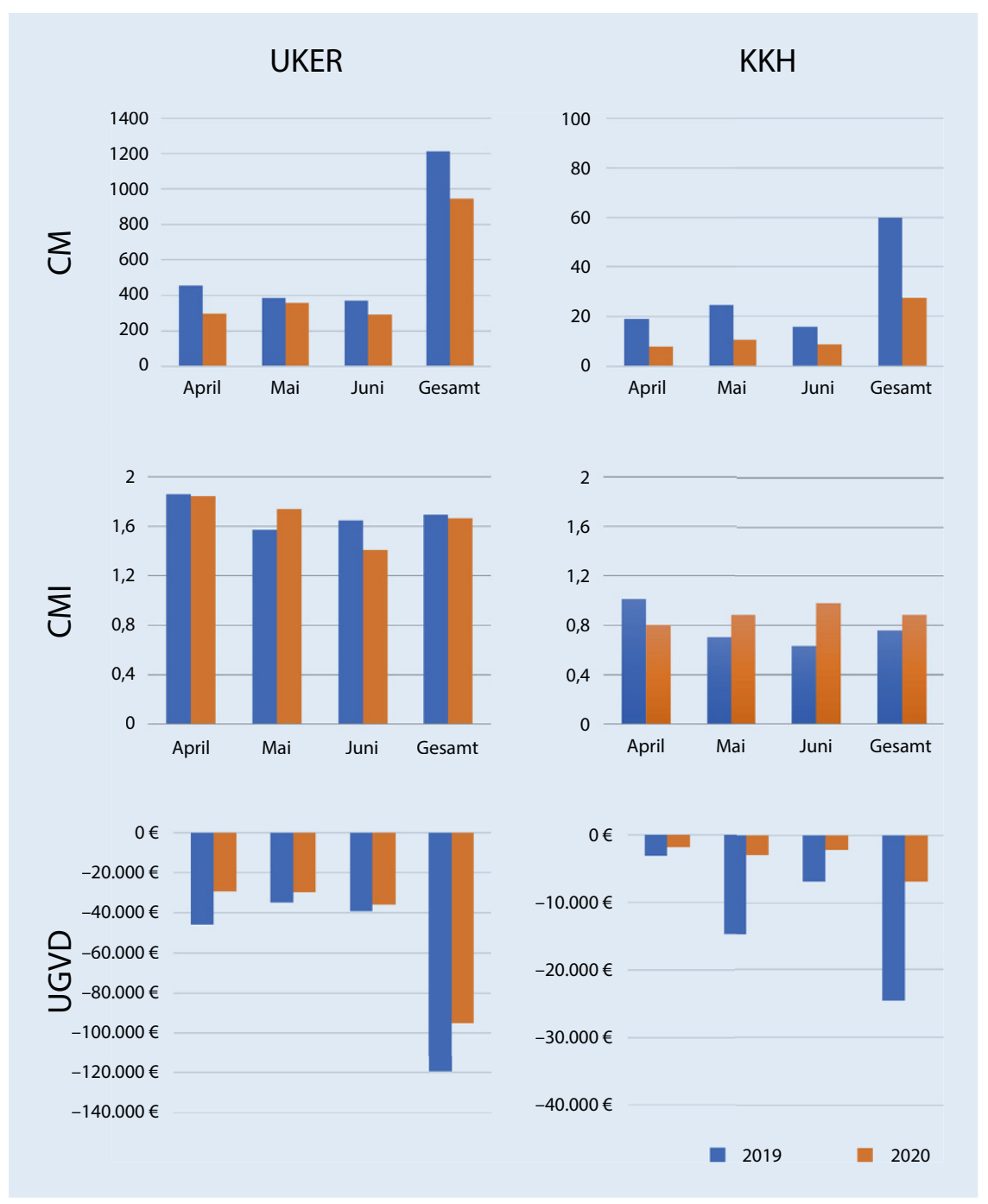

Abb. 1 \ Case Mix (CM), Case-Mix-Index (CMI) und untere Grenzverweildauer (UGVD) in der Klinik für Allgemein- und Viszeralchirurgie des Universitätsklinikums Erlangen (UKER) und in der Chirurgischen Abteilung des Kreiskrankenhauses St. Anna Höchstadt/Aisch (KKH) in den Jahren 2019 und 2020

dass es während der COVID-19-Pandemie im Frühjahr 2020 in Deutschland einen signifikanten Rückgang medizinischer Notfälle gab [21]. Entsprechend dieser Entwicklung fand sich in einer aktuellen populationsbasierten Analyse in Deutschland eine signifikant geringere Anzahl von Patienten mit unkomplizierter Blinddarmentzündung. Diese Studie zeigte jedoch auch, dass sich die Häufigkeit, die Behandlungsrate und die Komplikationsrate der komplizierten Blinddarmentzündung nicht unterschied [18]. Inwieweit eine Reduktion notfallmäßiger Krankenhausaufnahmen und Eingriffe tatsächlich zu einer Einschränkung der Gesundheitsversorgung bzw. zu Gesundheitsgefährdung in Deutschland geführt hat, bleibt an dieser Stelle unklar.

Im Laufe der Pandemie wurde deutlich, dass Patienten auch aufgrund von Ängsten vor einer Ansteckung mit SARS-CoV-2 (, severe acute respiratory syndrome coronavirus type $2^{\prime \prime}$ ) medizinische Einrichtungen meiden. Eine Folge der COVID-19-Pandemie könnte somit sein, dass onkologische Erkrankungen nicht oder erst in einem späteren Stadium entdeckt werden $[13,17]$. Zudem könnte es aufgrund von Vermeidungsstrategien dazu kommen, dass weniger aggressive (effektivere) Therapieoptionen bevorzugt empfohlen werden [15]. Solche Entwicklungen wären jedoch hochproblematisch, da diese mit einer signifikanten Reduktion an gewonnenen Le- bensjahren einhergehen [23]. In den von uns betrachteten Kliniken konnten wir keine Hinweise auf eine Therapieverzögerung finden. Laut einer nationalen Umfrage gaben jedoch $34 \%$ der Befragten an, dass sie aufgrund der Einschränkung des Normalbetriebs auch Verzögerungen in der onkologischen kolorektalen Chirurgie erwarten [2]. Diese Befürchtungen wurden später in einer bundesweiten Erhebung der Eingriffszahlen durch den Expertenbeirat des Bundesgesundheitsministeriums untermauert. Letzterer wies einen Rückgang von $12 \%$ im ersten Halbjahr 2020 nach [1].

Die Einschränkung des Krankenhausregelbetriebs und der Operationskapazitäten sollte initial vor allem der Schaffung von Intensivkapazitäten zur Behandlung von COVID-19-Patienten dienen. Da diese Patienten im Rahmen des COVID-19assoziierten Lungenversagens $u$.a. hochkomplexe intensivmedizinische Krankheitsbilder entwickeln, ist eine suffiziente Behandlung oft nur in spezialisierten Einrichtungen mit einer geeigneten Infrastruktur möglich. Vor diesem Hintergrund soll an dieser Stelle die Effizienz der bundesweiten, nichtselektiven Reduktion von Operationskapazitäten im stationären Sektor anhand der vorliegenden Daten hinterfragt werden. So sanken im betrachteten Zeitraum die Fallzahlen im KKH im Vergleich prozentual deutlich stärker. Somit konnte hier im Gegensatz zum Universitätsklinikum relativ gesehen eine höhere Entlastung intensivmedizinischer Behandlungskapazitäten erreicht werden. Da trotz moderner Ausstattung in Höchstadt dennoch die intensivmedizinischen Möglichkeiten nicht vergleichbar mit denen eines Universitätsklinikums sind, mussten im Verlauf COVID19-Intensivpatienten verlegt werden (z. B. fehlende Möglichkeit einer extrakorporalen Membranoxygenierung [ECMO] etc.). Demgegenüber blieb die Anzahl der onkologischen Operationen am UKER mit exzellenter intensivmedizinischer Infrastruktur unverändert (bundesweit konnte sogar eine leichte Zunahme an hochkomplexen Krebsoperationen registriert werden [1]). Dies führte wahrscheinlich dazu, dass wiederum Kapazitäten auf den Intensivstationen gebunden waren und damit dem initialen Ziel der Schaffung 
Tab. 3 Zusammensetzung der Brutto-und Nettoerlöse der Chirurgischen Kliniken Erlangen und Höchststadt in den Beobachtungszeiträumen 2019 und 2020

\begin{tabular}{|l|l|l|l|}
\hline \multicolumn{2}{|l|}{} & Erlangen & Höchststadt \\
\hline Erlöse aus DRG & 2019 & $4.323 .083 €$ & $212.450 €$ \\
\cline { 2 - 4 } & 2020 & $3.366 .615 €$ & $97.737 €$ \\
\hline Differenz zu 2019 & - & $-22,1 \%$ & $-54,0 \%$ \\
\hline Bruttoerlöse ${ }^{\mathrm{a}}$ & 2019 & $4.323 .083 €$ & $212.450 €$ \\
\hline Bruttoerlöse & 2020 & $4.012 .506 €$ & $209.047 €$ \\
\hline Erlöse aus DRG & $3.366 .615 €$ & $97.737 €$ \\
\hline Freihaltepauschale & $609.991 €$ & $109.564 €$ \\
\hline Zulagen & - & $35.900 €$ & $1.550 €$ \\
\hline Differenz zu 2019 & 2019 & $-7,2 \%$ & $-1,6 \%$ \\
\hline Nettoerlöse & 2020 & $4.323 .083 €$ & $212.450 €$ \\
\hline Nettoerlöse & $4.155 .977 €$ & $226.254 €$ \\
\hline Bruttoerlöse & $4.012 .507 €$ & $209.047 €$ \\
\hline Einsparungen aus variablen Sachkosten & $143.470 €$ & $17.207 €$ \\
\hline Differenz zu 2019 & - & $-3,9 \%$ & $6,5 \%$ \\
\hline $\begin{array}{l}\text { DRG „diagnosis-related groups" } \\
\text { aEntspricht den Erlösen aus DRG 2019 }\end{array}$ \\
\hline
\end{tabular}

von Intensivkapazitäten entgegenstanden. In den unterschiedlichen Phasen der Pandemie gab es auch unterschiedliche Auslastungen der Intensivstationen. Im Falle einer Überbelastung müssen alle freiwerdenden Kapazitäten auch in kleineren Krankenhäusern genutzt werden. Während Phasen der Entspannung bei sinkender Auslastung sollte in den Augen der Autoren die intensivmedizinische Behandlung schwerkranker COVID-19Patienten zur Sicherung einer optimalen Versorgung, wenn möglich, in Krankenhäusern der Maximalversorgung erfolgen. Somit könnte die ungezielte Einschränkung der Operationskapazitäten hier aus den genannten Gründe fehlgeleitet sein.

Auch wenn die flächendeckende, ungezielte Einschränkung des Krankenhausregelbetriebs und der Operationskapazitäten nicht effizient war, so war sie dennoch effektiv. Ab der 12. Kalenderwoche sank die durchschnittliche Anzahl von Patienten auf Intensivstationen in Deutschland [1]. Zudem zeigen die vorliegenden Daten, dass die Einschränkungen auch ohne eine negative Beeinflussung der Behandlungsqualität (gemessen an Surrogatparametern wie z. B. Verweildauer, Wiederaufnahmerate und Mortalitätsrate) vollzogen werden können.

Zu Beginn der Pandemie führten Stöß et al. eine nationale Umfrage unter den Ordinarien der deutschen Universitätskliniken der Allgemein- und Viszeralchirurgie durch [22]. Die geschätzten Veränderungen der Case-Mix-Punkte und der Erlöse wurden durchschnittlich mit $-26 \% \pm 12 \%$ bzw. $-28 \pm 12 \%$ angegeben, was jeweils etwas höher ist als der in Erlangen beobachtete Rückgang. Die befragten Ordinarien gingen zudem von einer deutlichen $\mathrm{Ab}$ nahme der Fallschwere (CMI $=-20 \pm 17 \%)$ aus. Eine logische Erklärung dieser Erwartung ergibt sich möglicherweise aus der Tatsache, dass Intensivbetten für chirurgische Patienten nur noch eingeschränkt zugänglich waren und somit komplexe Operationen mit einem höheren Risiko für eine mögliche Intensivpflicht nicht in unveränderter Frequenz durchgeführt werden konnten. In Erlangen blieb der CMI unverändert. Diese Stabilität der Fallschwere lässt sich unter anderem auf einen relativ hohen Anteil an onkologischen und notfallmäßigen Eingriffen zurückführen (zusammen fast $50 \%$ aller Eingriffe). In diesem Kontext ist die Entwicklung der bundesweiten Zahlen viszeralchirurgischer Operationen interessant. Wie oben bereits erwähnt, lässt sich hier eine gewisse Priorisierung hochkomplexer Eingriffe erkennen [1]. Eine Priorisierung hochkomplexer Eingriffe könnte für Kliniken höherer Versorgungsstufen aber auch eine Möglichkeit zur Stabilisierung von CMI und Erlösen sein. Auf diese Weise erfüllen die Kliniken dann zwar die gesundheitspolitischen Vorgaben zur Reduktion von Operationskapazitäten, erreichen aber mit ho- her Wahrscheinlichkeit nicht eine maximale Entlastung der Intensivstationen. Im Vergleich zu der erwarteten und beobachteten Entwicklung der Erlöskennzahlen im universitären Maximalversorger reduzierten sich der CM und die DRG-Erlöse im Grundversorger Höchstadt um 54\%. Der CMI des KKH blieb statistisch unverändert. An dieser Stelle zeigt sich, dass kleine Krankenhäuser - wie das KKH - ohne die finanziellen Hilfen des COVID-19-Krankenhausentlastungsgesetzes besonders stark von der Einschränkung des Krankenhausregelbetriebs getroffen werden.

Die Zusammensetzung der Brutto- und Nettoerlöse (• Tab. 3) zeigt, dass die chirurgische Klinik des Krankenhauses der unteren Versorgungsstufe zu Beginn der Pandemie möglicherweise überproportional vergütet wurde. Legt man die abgestuften Freihaltepauschalen unseren Berechnungen zugrunde, so zeigt sich für beide Versorgungsstufen eine wesentlich ausgeglichenere Verteilung der Hilfen. Die Anpassungen der fixen Freihaltepauschale sind somit als wichtige Entscheidung zu werten. Der Expertenrat sah jedoch noch im August 2020 eine „Verlängerung der finanziellen Hilfen nach § 21 KHG [...] über den 30. September 2020 hinaus [...]" als nicht erforderlich an. Vielmehr solle ein individueller Ausgleich mit den Kostenträgern vereinbart werden [4]. Aufgrund der aktuellen zweiten Pandemiewelle wurden die abgestuften Freihaltepauschalen für den Zeitraum vom 18.11.2020 bis einschließlich 31.01.2021 wieder eingeführt. Finanzielle Hilfen für Krankenhäuser sind demnach weiter vorgesehen. Die Auszahlung erfolgt jedoch gezielter anhand der 7-Tages-Inzidenz ( $>70$ ) sowie der Zahl der freien Intensivbetten ( $<25 \%)$ an den Krankenhäusern, die mindestens die Notfallstufe 2 abdecken müssen [5, 16]. Da viele Krankenhäuser aktuell unter einem starken wirtschaftlichen Druck stehen, wird dieses Vorgehen bereits kritisiert [9, 12, 19]. Im Hinblick auf die fehlende Effizienz der flächendeckenden Einschränkung des Regelbetriebs erscheint eine Selektion der Auszahlung und somit eine gezielte, bedarfsgerechte Einschränkung jedoch wünschenswert. 


\section{Originalien}

\section{Limitationen}

Die Ergebnisse der Studie sind unter Berücksichtigung einiger Limitationen zu betrachten. Aufgrund der fachspezifischen Auswahl mit Auswertung von lediglich zwei Kliniken sind die Ergebnisse nicht repräsentativ für das Fachgebiet und auch nicht übertragbar auf das deutsche Gesundheitssystem als Ganzes. Hierfür sollte eine groß angelegte retrospektive Analyse der Abrechnungsdaten erfolgen. Da keine Daten zu ambulanten Einweisungen vorliegen, kann auch keine Aussage über eine Dynamik in diesem Bereich getroffen werden. Die Beurteilung der Sachkosten fand vereinfacht nach der etablierten Methode des Expertenrats statt. Daher können mögliche Änderungen der Kostenstruktur auch hier nicht detailliert abgebildet werden (z. B. durch Reduktion teurer Technologien o. Ä.). Eine Aussage über die intensivmedizinische Behandlung von COVID19-Patienten sollte und kann aus dieser Studie ebenfalls nicht getroffen werden.

\section{Schlussfolgerung}

Während der ersten Pandemiewelle hat die bundesweite Einschränkung des Regelbetriebs zu einer effektiven Schaffung von Intensivkapazitäten geführt. Die Effizienz dieser ungezielten Maßnahme sollte jedoch, im Hinblick auf eine optimale Versorgungsqualität, hinterfragt werden. Darüber hinaus könnte die fixe Freihaltepauschale mit einer ungerechten Verteilung zwischen chirurgischen Kliniken unterschiedlicher Versorgungsstufen verbunden sein. Die Einführung der abgestuften Freihaltepauschalen führt möglicherweise auch in der Allgemein- und Viszeralchirurgie zu einer höheren Verteilungsgerechtigkeit. Trotz dieser Anpassung können ohne eine individuelle Berücksichtigung der lokalen Gegebenheiten jedoch Finanzierungsengpässe entstehen. 


\section{Anhang}

\begin{tabular}{|c|c|c|c|c|c|c|c|c|c|}
\hline \multirow[t]{2}{*}{ Indikationsart } & \multirow[t]{2}{*}{ Operationskategorien } & \multicolumn{2}{|c|}{ Jahr (UKER) } & \multirow[t]{2}{*}{ Differenz } & \multirow{2}{*}{$\begin{array}{l}\text { Ge- } \\
\text { samt }\end{array}$} & \multicolumn{2}{|c|}{ Jahr (KKH) } & \multirow[t]{2}{*}{ Differenz } & \multirow{2}{*}{$\begin{array}{l}\text { Ge- } \\
\text { samt }\end{array}$} \\
\hline & & 2019 & 2020 & & & 2019 & 2020 & & \\
\hline \multirow[t]{19}{*}{ Elektiv } & Adipositaschirurgie & 11 & 7 & $-36,36 \%$ & 18 & - & - & - & 0 \\
\hline & Appendektomie & 2 & 0 & $-100,00 \%$ & 2 & - & - & - & 0 \\
\hline & Cholezystektomie & 17 & 13 & $-23,53 \%$ & 30 & 4 & 2 & $-50,00 \%$ & 6 \\
\hline & Dünndarmresektion & 4 & 6 & $50,00 \%$ & 10 & - & - & - & 0 \\
\hline & Endokrine Chirurgie & 17 & 3 & $-82,35 \%$ & 20 & - & - & - & 0 \\
\hline & Hepatobiliäre Chirurgie & 17 & 23 & $35,29 \%$ & 40 & - & - & - & 0 \\
\hline & Hernienchirurgie & 27 & 12 & $-55,56 \%$ & 39 & 24 & 14 & $-41,67 \%$ & 38 \\
\hline & Kolorektale Chirurgie & 87 & 52 & $-40,23 \%$ & 139 & 1 & 0 & $-100,00 \%$ & 1 \\
\hline & Lymphadenektomie & 3 & 11 & $266,67 \%$ & 14 & - & - & - & 0 \\
\hline & Melanom Chirurgie & 29 & 21 & $-27,59 \%$ & 50 & - & - & - & 0 \\
\hline & Milz & 3 & 3 & $0,00 \%$ & 6 & - & - & - & 0 \\
\hline & Multiviszerale Resektion & 2 & 4 & $100,00 \%$ & 6 & - & - & - & 0 \\
\hline & Ösophagus-/Magenchirurgie & 30 & 14 & $-53,33 \%$ & 44 & - & - & - & 0 \\
\hline & Pankreaschirurgie & 27 & 15 & $-44,44 \%$ & 42 & - & - & - & 0 \\
\hline & Proktologie & 105 & 57 & $-45,71 \%$ & 162 & 12 & 4 & $-66,67 \%$ & 16 \\
\hline & Sarkomchirurgie & 19 & 19 & $0,00 \%$ & 38 & - & - & - & 0 \\
\hline & Sonstiges & 11 & 15 & $36,36 \%$ & 26 & 10 & 4 & $-60,00 \%$ & 14 \\
\hline & Stomaanlage/Resektion & 37 & 25 & $-32,43 \%$ & 62 & 3 & 2 & $-33,33 \%$ & 3 \\
\hline & Gesamt & 448 & 300 & $-33,04 \%$ & 748 & 54 & 26 & $-51,85 \%$ & 81 \\
\hline \multirow[t]{14}{*}{ Notfallmäßig } & Appendektomie & 37 & 35 & $-5,41 \%$ & 72 & 3 & 2 & $-33,33 \%$ & 4 \\
\hline & Cholezystektomie & 19 & 20 & $5,26 \%$ & 39 & 1 & 1 & $0,00 \%$ & 2 \\
\hline & Dünndarmresektion & 11 & 17 & $54,55 \%$ & 28 & 1 & 0 & $-100,00 \%$ & 1 \\
\hline & Hepatobiliäre Chirurgie & 1 & 0 & $-100,00 \%$ & 1 & - & - & - & 0 \\
\hline & Hernienchirurgie & 9 & 6 & $-33,33 \%$ & 15 & 4 & 0 & $-100,00 \%$ & 4 \\
\hline & Kolorektale Chirurgie & 21 & 17 & $-19,05 \%$ & 38 & 1 & 0 & $-100,00 \%$ & 1 \\
\hline & Milz & 0 & 3 & $300,00 \%$ & 3 & - & - & - & - \\
\hline & Ösophagus-/Magenchirurgie & 4 & 3 & $-25,00 \%$ & 7 & - & - & - & - \\
\hline & Pankreaschirurgie & 1 & 1 & $0,00 \%$ & 2 & - & - & - & - \\
\hline & Proktologie & 39 & 35 & $-10,26 \%$ & 74 & - & - & - & - \\
\hline & Sarkomchirurgie & 2 & 2 & $0,00 \%$ & 4 & - & - & - & - \\
\hline & Sonstiges & 19 & 24 & $26,32 \%$ & 43 & 13 & 6 & $-53,85 \%$ & 19 \\
\hline & Stomaanlage/Resektion & 12 & 9 & $-25,00 \%$ & 21 & - & - & - & 0 \\
\hline & Gesamt & 175 & 172 & $-1,71 \%$ & 347 & 23 & 8 & $-60,87 \%$ & 31 \\
\hline
\end{tabular}


Funding. Open Access funding enabled and organized by Projekt DEAL.

\section{Einhaltung ethischer Richtlinien}

Interessenkonflikt. J. Binder, M. Brunner, M. Maak, A. Denz, G. F. Weber, R. Grützmann und C. Krautz geben an, dass kein Interessenkonflikt besteht.

Für diesen Beitrag wurden von den Autoren keine Studien an Menschen oder Tieren durchgeführt. Für die aufgeführten Studien gelten die jeweils dort angegebenen ethischen Richtlinien.

\section{Korrespondenzadresse}

\section{PD Dr. med. Christian Krautz}

Klinik für Allgemein- und Viszeralchirurgie des, Universitätsklinikum Erlangen Krankenhausstraße 12, Eingang Maximiliansplatz, 91054 Erlangen, Deutschland christian.krautz@uk-erlangen.de

Open Access. Dieser Artikel wird unter der Creative Commons Namensnennung 4.0 International Lizenz veröffentlicht, welche die Nutzung, Vervielfältigung, Bearbeitung, Verbreitung und Wiedergabe in jeglichem Medium und Format erlaubt, sofern Sie den/die ursprünglichen Autor(en) und die Quelle ordnungsgemäß nennen, einen Link zur Creative Commons Lizenz beifügen und angeben, ob Änderungen vorgenommen wurden.

Die in diesem Artikel enthaltenen Bilder und sonstiges Drittmaterial unterliegen ebenfalls der genannten Creative Commons Lizenz, sofern sich aus der Abbildungslegende nichts anderes ergibt. Sofern das betreffende Material nicht unter der genannten Creative Commons Lizenz steht und die betreffende Handlung nicht nach gesetzlichen Vorschriften erlaubt ist, ist für die oben aufgeführten Weiterverwendungen des Materials die Einwilligung des jeweiligen Rechteinhabers einzuholen.

Weitere Details zur Lizenz entnehmen Sie bitte der Lizenzinformation auf http://creativecommons.org/ licenses/by/4.0/deed.de.

\section{Literatur}

1. Augurzky B, Busse R, Haering A, Nimptsch U, Pilny A, Werbeck A (2020) Analysen zum Leistungsgeschehen, zur Erlössituation von Krankenhäusern und zu betroffenen Patienten und ihrer Versorgung in der Corona-Krise, in: Bundesministerium für Gesundheit, S. 1-29. https://www.bundesgesundheitsministerium.de/ ministrium/meldungen/2020/abschlussberichtdes-expertenbeirats-zu-den-auswirkungen-descovid-19-krankenhausentlastungsgesetzes.html. Zugegriffen:5.Okt. 2020

2. Brunner M, Krautz C, Kersting S, Weber GF, Stinner B, Benz SR, Grützmann R (2020) Oncological colorectal surgery during the COVID-19 pandemic-a national survey. J Int Colorectal Dis :1-7. https:// doi.org/10.1007/s00384-020-03697-6

3. Bundesministerium für Gesundheit (BMG), Referat L7 „Presse, Internet, Soziale Netzwerke”

Economic impact of the COVID-19 pandemic in general and visceral surgery. A comparison of performance and revenue data from two departments of hospitals with different levels of referral

Background: During the first wave of the coronavirus disease 2019 (COVID-19) pandemic, German hospitals were required to limit the capacity for elective surgery to prevent the healthcare system from general overload. In March 2020, the German government passed the COVID-19 Hospital Relief Act that guaranteed compensation payments for these limitations. In this study the regional impact of this intervention were analyzed.

Material and methods: The performance data and revenue figures for the departments of general and visceral surgery of the University Hospital of Erlangen (UKER) and the District Hospital St. Anna Höchstadt/Aisch (KKH) during the period from 1 April to 30 June 2019 were compared with the respective period in 2020.

Results: There was a significant decrease in bed occupancy rates and case numbers of inpatient treatment. The latter declined by $20.06 \%$ in the UKER and $60.76 \%$ in the KKH. Nononcological elective surgery was reduced by $33.04 \%$ in the UKER and $60.87 \%$ in the KKH. The number of emergency procedures remained unchanged in the UKER, while they decreased by $51.58 \%$ in the $\mathrm{KKH}$. The revenues from diagnosis-related groups (DRG) decreased by $22.12 \%$ (UKER) and $54 \%$ (KKH), respectively. After taking compensation payments and savings from variable material costs into account, the UKER recorded a loss of $-3.87 \%$, while there was a positive revenue effect of $6.5 \%$ in the KKH.

Discussion: The nonselective restriction of elective surgery had a significant impact on patient care and revenue figures at both locations. With respect to the increase of intensive care capacities, such untargeted measures do not appear to be efficient. In addition, the fixed rate of compensation payments led to an unbalanced distribution of the financial aid between the two departments.

\section{Keywords}

Hospital Relief Act · Coronavirus · Surgery · DRG billing data - Treatment quality

(2020) Coronavirus SARS-CoV-2: Chronik der bisherigen Maßnahmen. URL: https://www. bundesgesundheitsministerium.de/coronavirus/ chronik-coronavirus.html. Zugegriffen: 29. Dez. 2020.

4. Bundesministerium für Gesundheit (2020) Abschlussberichtzur Überprüfung der Auswirkungen der Regelungen in den $\$ \S 21$ bis 23 auf die wirtschaftliche Lage der Krankenhäuser durch den Beirat gemäß § 24 Krankenhausfinanzierungsgesetz. https://www.bundesgesundheitsministerium. de/fileadmin/Dateien/3_Downloads/K/200821_ Abschlussbericht_des_Beirats_gemaess__24_ KHG_Abschlussbericht.pdf. Zugegriffen: 27. Dez. 2020

5. Bundesministerium für Justiz und Verbraucherschutz (2020) Gesetz zur wirtschaftlichen Sicherung der Krankenhäuser und zur Regelung der Krankenhauspflegesätze (Krankenhausfinanzierungsgesetz - KHG) § 21 Ausgleichszahlungen an Krankenhäuser aufgrund von Sonderbelastungen durch das neuartige Coronavirus SARS-CoV-2. http://www.gesetze-im-internet.de/khg/_21. html.Zugegriffen:29. Dez. 2020

6. COVID-19-Krankenhausentlastungsgesetz vom 27. März 2020 (BGBI. 2020|580).

7. COVID-19-Ausgleichszahlungs-Anpassungs-Verordnung - AusgIZAV vom 3. Juli 2020 (BGBI. 2020| 1556).

8. von Dercks N, Seehofer D, Steinert M, Krämer S, Branzan D, Dietrich A, Schürmann O, Gockel I (2020) Wie stark trifft die Corona-Pandemie die chirurgische Klinik eines universitären Maximal- versorgers?: Eine Analyse der ersten 7 Wochen [How severe is the effect of the coronavirus pandemic on the department of surgery of a university hospital? An analysis of the first 7 weeks]. Chirurg 91(9):755-761

9. Deutsche Krankenhausgesellschaft (2020) Dringender Appell der Deutschen Krankenhausgesellschaft und aller Landeskrankenhausgesellschaften an die Bundesregierung und die Regierungen in den Ländern zur Lösung der wirtschaftlichen Probleme der deutschen Krankenhäuser und Sicherstellung der Patientenversorgung in der aktuellen Corona-Pandemie. URL: https://www.dkgev. de/fileadmin/default/Mediapool/1_DKG/1.7 Presse/1.7.1_Pressemitteilungen/2020/Appell_ zur_Loesung_der_wirtschaftlichen_Probleme_ der_Krankenhaeuser_fuer_das_Gesamtjahr_ 2021.pdf.Zugegriffen:29. Dez. 2020.

10. Deutscher Evangelischer Krankenhausverband e.V. (2020) Vier-Punkte-CoronaSofortprogramm Krankenhäuser. https:// dekv.de/positionen/vier-punkte-coronasofortprogramm-krankenhaeuser. Zugegriffen:29.Dez. 2020

11. Deutsches Ärzteblatt (2020) Coronakrise: Krankenhäusern drohen Ausfälle in Millionenhöhe. https://www.aerzteblatt.de/nachrichten/ 111121/Coronakrise-Krankenhaeusern-drohenAusfaelle-in-Millionenhoehe. Zugegriffen: 29. Dez. 2020

12. Deutsches Ärzteblatt (2020) Mehr Krankenhäuser sollen Freihaltepauschalen erhalten können. https://www.aerzteblatt.de/ 
nachrichten/119556/Mehr-Krankenhaeusersollen-Freihaltepauschalen-erhalten-koennen. Zugegriffen:29.Dez. 2020

13. Dinmohamed AG, Visser O, Verhoeven RHA, Louwman MWJ, van Nederveen FH, Willems SM, Merkx MAW, Lemmens VEPP, Nagtegaal ID, Siesling S (2020) Fewer cancer diagnoses during the COVID-19 epidemic in the Netherlands. Lancet Oncol 21(6):750-751. https://doi.org/10.1016/S1470-2045(20)30265-5 (Erratum in: Lancet Oncol. 2020 May 4;: PMID: 32359403)

14. Flintrop J (2020) Streit um Krankenhausrechnungen: Der Gesetzgeber soll es richten. Dtsch Arztebl 108(42):A-2190 / B-1850/C-1830 (https:// www.aerzteblatt.de/archiv/110443/Streit-umKrankenhausrechnungen-Der-Gesetzgeber-solles-richten.Zugegriffen: 10.12.2020)

15. Fröhlich S, Arndt V (2020) Versorgung von Krebspatienten: Corona-Effekt in der Onkologie. Dtsch Arztebl 117(46):A-2234/B-1893

16. Spitzenverband GKV (2020) Prognose der Notfallstufen, Stand 14.12.2020. https://www. gkv-spitzenverband.de/media/dokumente/ krankenversicherung_1/krankenhaeuser/KH Corona_Prognose_der_Notfallstufen_Stand_1412-2020.pdf.Zugegriffen:29. Dez. 2020

17. Jones D, Neal RD, Duffy SRG, Scott SE, Whitaker KL, Brain K (2020) Impact of the COVID-19 pandemic on the symptomatic diagnosis of cancer: the view from primary care. Lancet Oncol 21(6):748-750. https://doi.org/10.1016/S1470-2045(20)30242-4

18. Köhler $F$, Acar L, van den Berg A, Flemming $S$, Kastner C, Müller S, Diers J, Germer CT, Lock JF, L'hoest $\mathrm{H}$, Marschall U, Wiegering A (2021) Impact of the COVID-19 pandemic on appendicitis treatment in Germany-a population-based analysis. Langenbecks Arch Surg. https://doi.org/ 10.1007/s00423-021-02081-4

19. RedaktionsNetzwerk Deutschland $\mathrm{GmbH}$ (2020) Krankenhausgesellschaft: Kliniken können bald Gehälter nicht mehr zahlen. https://www.rnd.de/politik/fehlende-gelderin-kliniken-bei-krankenhaus-mitarbeiterndrohen-engpasse-bei-den-gehaltszahlungen7WOFZHNQCBHTJDQLZBI6JZB5E4.html. Zugegriffen:29. Dez. 2020

20. Robert Koch Institut (2020) COVID-19: Fallzahlen in Deutschland und weltweit, Fallzahlen in Deutschland. https://www.rki.de/DE/Content/InfAZ/N/ Neuartiges_Coronavirus/Fallzahlen.html. Zugegriffen:24. Febr. 2021

21. Slagman $A$, Behringer $W$, Greiner $F$, Klein $M$ Weismann D, Erdmann B, Pigorsch M, Möckel M (2020) Medical emergencies during the COVID-19 pandemic. An analysis of emergency department data in Germany. Dtsch Arztebl Int 117:545-552. https://doi.org/10.3238/arztebl.2020.0545

22. StößC, Haffer $H$, Steffani M, Pergolinil, Hartmann $D$, Nitsche U, Novotny A, Friess $H$, Müller MW (2020) Effects of the SARS-CoV-2 pandemic on surgery - a national cross-sectional study. Chirurg 91(9):762-768

23. Sud A, Jones ME, Broggio J, Loveday C, Torr B, Garrett A, Nicol DL, Jhanji S, Boyce SA, Gronthoud F, Ward P, Handy JM, Yousaf N, Larkin J, Suh YE, ScottS, Pharoah P, Swanton C, Abbosh C, Williams M, Lyratzopoulos G, Houlston RTurnbull C (2020) Collateral damage: the impact on outcomes from cancer surgery of the COVID-19 pandemic. Ann Oncol31(8):1065-1074. https://doi.org/10.1016/j. annonc.2020.05.009

24. Verband der Universitätsklinika Deutschlands e.V. (VUD) (2019) Vorläufige Stellungnahme zum
Referentenentwurf eines Gesetzes für bessere und unabhängigere Prüfungen - MDK-Reformgesetz, in: Bundesministerium für Gesundheit S. 1-13. https://www.bundesgesundheitsministerium.de/ fileadmin/Dateien/3_Downloads/Gesetze_und_ Verordnungen/Stellungnahmen_WP19/MDK/ VUD.pdf.Zugegriffen:27.Dez.2020
Hier steht eine Anzeige. Springer 